\title{
Multidisciplinary approach to rare primary cardiac sarcoma: a case report and review
}

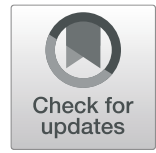

\author{
Audrone Vaitiekiene ${ }^{1 *}$, Domas Vaitiekus ${ }^{2}$, Laura Urbonaite ${ }^{1}$, Antanas Jankauskas ${ }^{3}$, Justina Portacenko ${ }^{1}$, \\ Tomas Lapinskas ${ }^{1}$, Rimantas Benetis ${ }^{4,5}$, Adakrius Siudikas ${ }^{4}$, Audrone Veikutiene ${ }^{4}$, Lina Poskiene ${ }^{6}$, \\ Ausra Kavoliuniene ${ }^{1}$, Rasa Janciauskiene ${ }^{2}$, Laimonas Jarusevicius ${ }^{2}$, Elona Juozaityte ${ }^{2}$, Remigijus Zaliunas ${ }^{1}$ and \\ Egle Ereminiene ${ }^{1}$
}

\begin{abstract}
Background: Undifferentiated pleomorphic sarcoma is a very rare and aggressive type of primary cardiac tumors. Most cardiac sarcomas result in rapid growth and quick death. According to different sources the median survival is typically 6 to 12 months. We are presenting a case of primary cardiac sarcoma with 26 months disease free survival following cytoreductive surgery and chemotherapy.

Case presentation: A 48-year-old woman with progressing symptoms of dyspnea and palpitations for over 2 months was referred to a cardiologist. With the help of echocardiography and cardiovascular magnetic resonance cardiac sarcoma was suspected. Open biopsy and cytoreductive surgery were performed, complete resection of the tumor was not possible. Histology revealed undifferentiated pleomorphic sarcoma. Seven cycles of chemotherapy with Doxorubicine and Ifosfamide were completed. Cardiovascular magnetic resonance revealed a complete response only signs of fibrosis without any signs of tumor were visible. Follow ups with echocardiography, cardiovascular magnetic resonance and chest, abdomen and pelvic computed tomography is performed every 3 months. Twenty-six months from initial diagnosis the patient is still free of recurrence of tumor with no compromises of the quality of life.
\end{abstract}

Conclusion: Standard chemotherapy together with cytoreductive surgery can have a complete response effect in undifferentiated pleomorphic sarcoma with unusual long-term survival.

Keywords: Cardiac sarcoma, Cardiovascular magnetic resonance, Undifferentiated pleomorphic sarcoma

\section{Background}

Undifferentiated pleomorphic sarcoma (UPS), also known as malignant fibrous histiocytoma is an extremely rare type of primary cardiac tumor $[1,2]$. According to a study conducted in Cleveland Clinic UPS were diagnosed in $12 \%$ of all cardiac sarcoma cases [3]. Clinical presentation varies, they can be symptomatic, imitating different cardiac conditions (symptoms depend on the localization of tumor, invasion into the myocardium, but not histology) or found incidentally on echocardiography, MRI or CT examinations. UPSs are aggressive and locally invasive tumors, frequently making complete surgical excision unfeasible, which leads to a poor prognosis and a low survival rate [4].

\footnotetext{
* Correspondence: audrone.vaitiekiene@lsmuni.It

1 Department of Cardiology, Medical Academy, Lithuanian University of Health Sciences, Kaunas, Lithuania

Full list of author information is available at the end of the article
}

Even with complete resection, most patients develop recurrent disease rapidly, thus according to different sources the median survival is typically 6 to 12 months [5]. We are presenting a case of primary cardiac sarcoma with 26 months disease free survival following cytoreductive surgery and chemotherapy.

\section{Case presentation}

A 48-year-old female with progressing symptoms of dyspnea and palpitations for over 2 months was referred to cardiologist. Her medical history was unremarkable, she only had allergy to various chemical substances as she was working as a tailor. Auscultation of the heart revealed a loud systolic murmur throughout entire precordium with irradiation to the left shoulder blade. Hemodynamic parameters were normal, while the ECG showed left ventricular hypertrophy (LVH). Laboratory findings revealed

(c) The Author(s). 2019 Open Access This article is distributed under the terms of the Creative Commons Attribution 4.0 International License (http://creativecommons.org/licenses/by/4.0/), which permits unrestricted use, distribution, and reproduction in any medium, provided you give appropriate credit to the original author(s) and the source, provide a link to the Creative Commons license, and indicate if changes were made. The Creative Commons Public Domain Dedication waiver (http://creativecommons.org/publicdomain/zero/1.0/) applies to the data made available in this article, unless otherwise stated. 
a normocytic normochromic anemia (Hb $104 \mathrm{~g} / \mathrm{l}$, normal values $119-146 \mathrm{~g} / \mathrm{l}$ ) and elevated level of lactate dehydrogenase (LDH) without liver or renal dysfunction.

Transthoracic echocardiography (TTE) was performed. Several large masses in the left ventricle (LV) close to anterior and anterolateral mid-ventricular and apical segments were observed with one tumor (approximately $2.0 \mathrm{~cm}$ in length) partially obstructing LV outflow tract. Additional smaller tumor was seen in left atrium (LA) attached to the interatrial septum (IAS).

Cardiovascular magnetic resonance (CMR) was performed to assess specific characteristics of masses using a $1.5 \mathrm{~T}$ scanner (Siemens Magnetom Aera, Siemens AG Healthcare Sector, Erlangen, Germany) with an 18channel phased array coil. Cine images (Fig. 1a) showed a $71 \times 45 \times 21 \mathrm{~mm}$ tumor with irregular borders in the LV attached to anterior and anterolateral walls. The mass was partially infiltrating LV myocardium and was isointense on non-contrast T1W spin-echo images (Fig. 1b). On T2W spin-echo images the tumor appeared hyperintense (Fig. 1c), early gadolinium enhancement was similar to myocardium. The tumor heterogeneously enhanced after administration of full dose of contrast agent (Fig. 1d). The diagnosis of malignant cardiac sarcoma was suspected. Also computed tomography (CT) of the chest and abdomen was performed, but there were no signs of other origin of malignancy or metastasis.

Open biopsy and cytoreductive surgery were performed. The masses from LV cavity, outflow tract and LA were removed, apical trabeculae were infiltrated by the tumor and complete resection was not possible. Histology revealed UPS. (Fig. 2) Cardiomyocytes were infiltrated with tumor cells. The tumor was composed of pleomorphic cells with abundant mitoses and area of necrosis. Focal positivity of CD34, CD99 and TLE-1 were not specific. Tumor cells were negative for actin, desmin, CD34, CD117, S100P, EMA and panCK immunomarkers.

Postoperative CMR revealed no masses in the LA and LV outflow tract. Tumor related to the LV cavity was still present (Fig. 3). Additionally, there was a pericardial effusion of $12 \mathrm{~mm}$ localized at LV inferior wall and right ventricle.
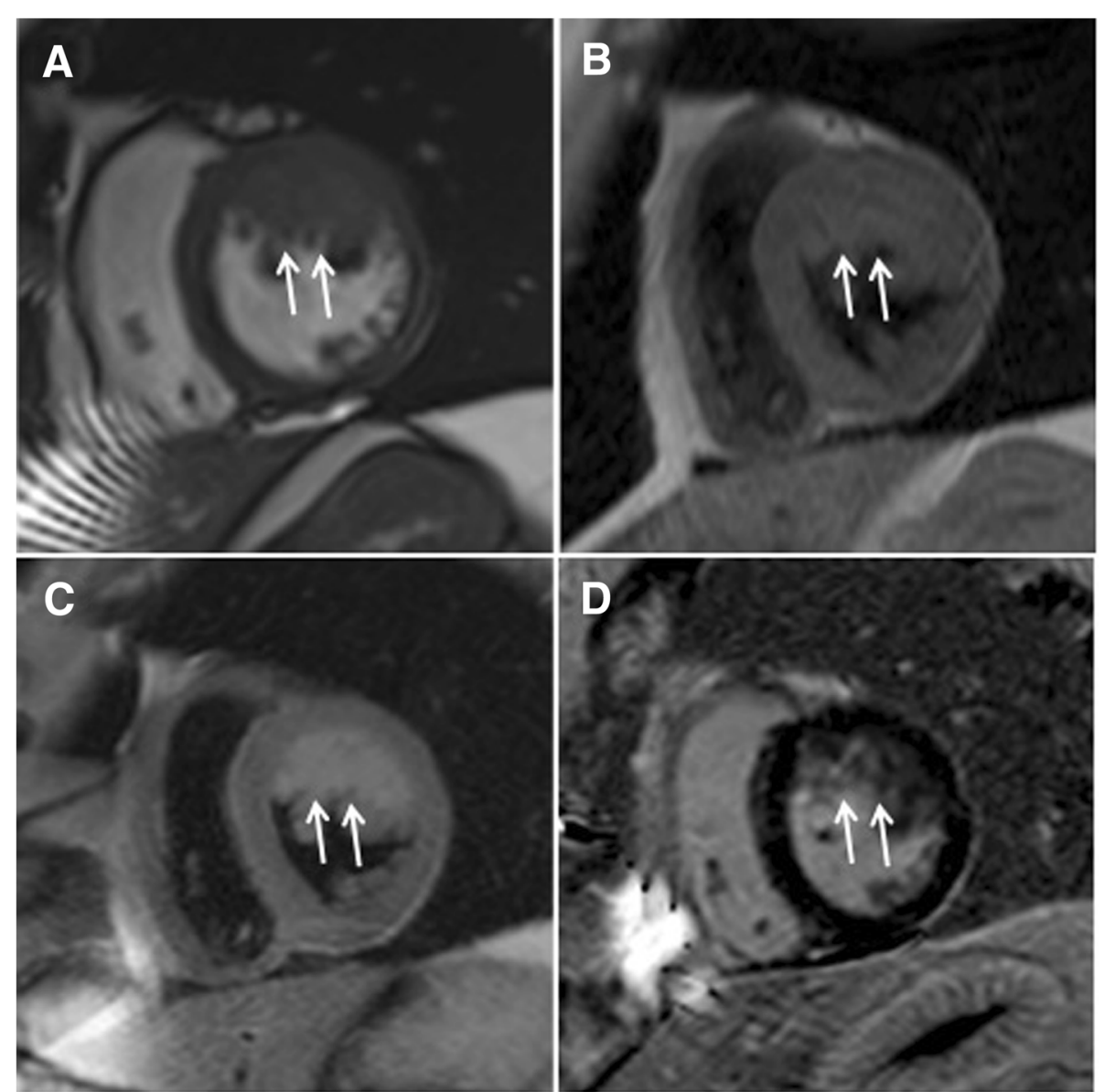

Fig. 1 CMR examination before surgery. The cine image (a) demonstrates tumor (arrows) in the LV adherent to the anterior and anterolateral walls. Mass (arrows) appears isointense in T1-weighted (b), hyperintense in T2-weighted (c) and heterogeneously enhanced in late gadolinium enhancement (d) images 


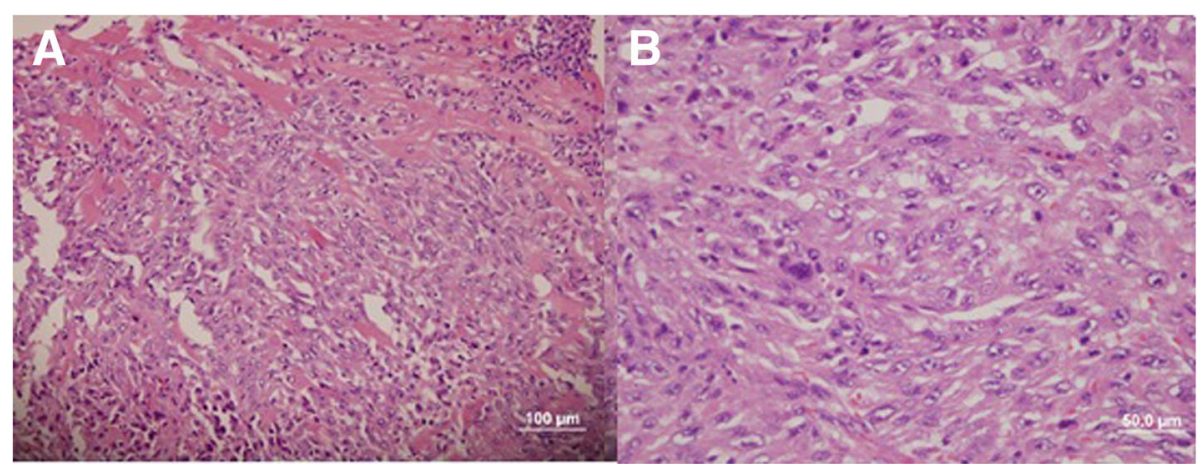

Fig. 2 Histological examination: undifferentiated pleomorphic sarcoma: a - infiltration among cardiomyocytes, b - cell morphology

Chemotherapy with doxorubicin and ifosfamide was initiated. The scheme was: ifosfamide $3 \mathrm{~g} / \mathrm{m}^{2} /$ day 1-3 days, Mesna $100 \%$ ifosfamide dose, doxorubicin $75 \mathrm{mg} / \mathrm{m}^{2} /$ day 1 day. Granulocyte-colony stimulating factors (GCSF) were given. According to CTCAE (Common Terminology Criteria for Adverse Events) only I grade neutropenia and thrombocytopenia were observed. Totally, 7 cycles were completed (total cumulative doxorubicin dose $525 \mathrm{mg}$ / $\mathrm{m}^{2}$ ). Echocardiography and CMR were performed to assess the results of chemotherapy treatment and revealed a complete response - only signs of fibrosis without any signs of tumor were visible in CMR images (Fig. 4). The chest and abdomen CT were repeated and did not demonstrate any findings suggesting metastasis.

Follow ups with TTE, CMR and chest, abdomen and pelvic CT is performed every 3 months. Twenty-six

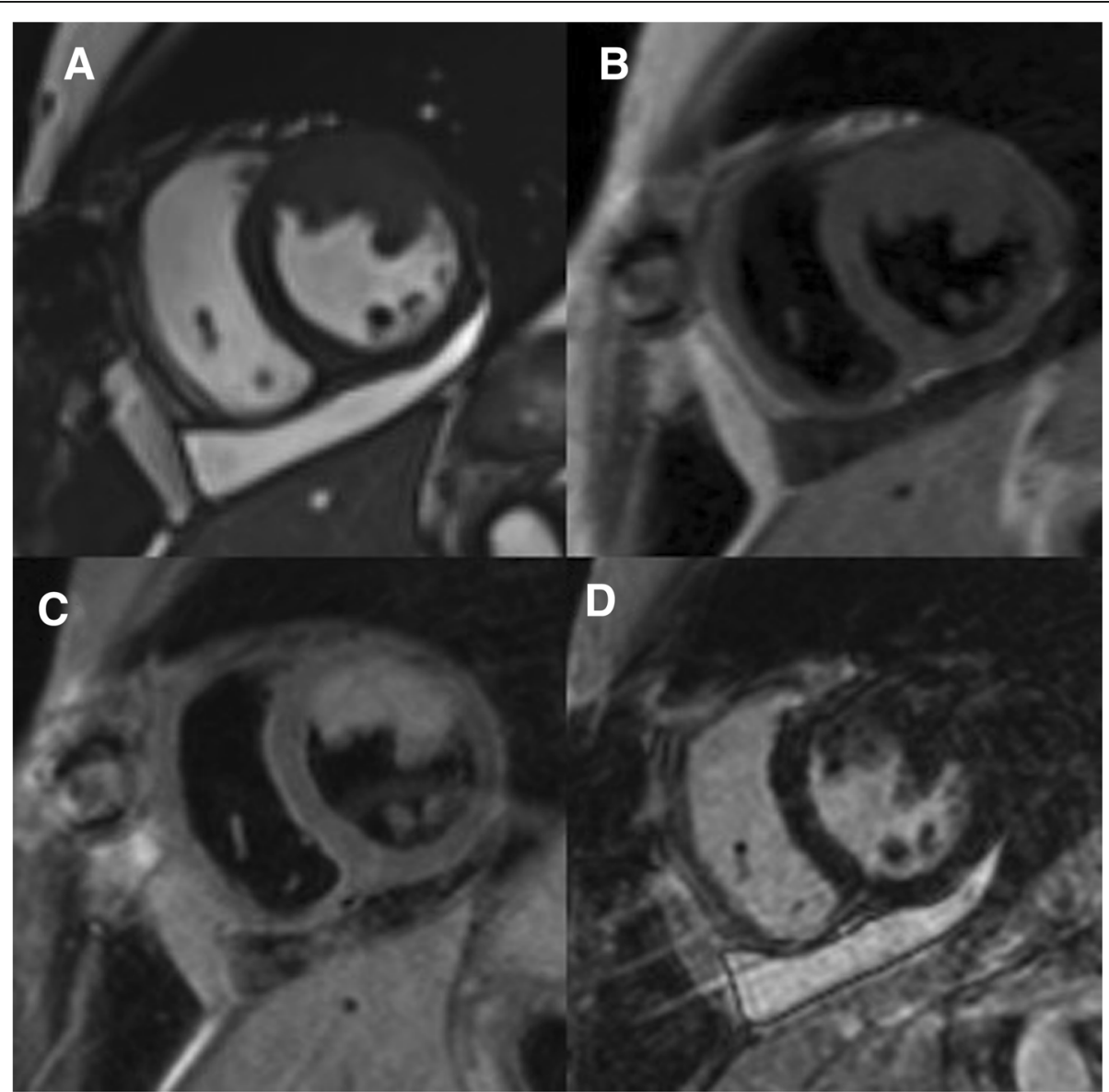

Fig. 3 Residual tumor masses after cytoreductive surgery in the anterior and lateral wall of LV in cine (a), T1-weighted (b), T2-weighted (c) and LGE (d) images 

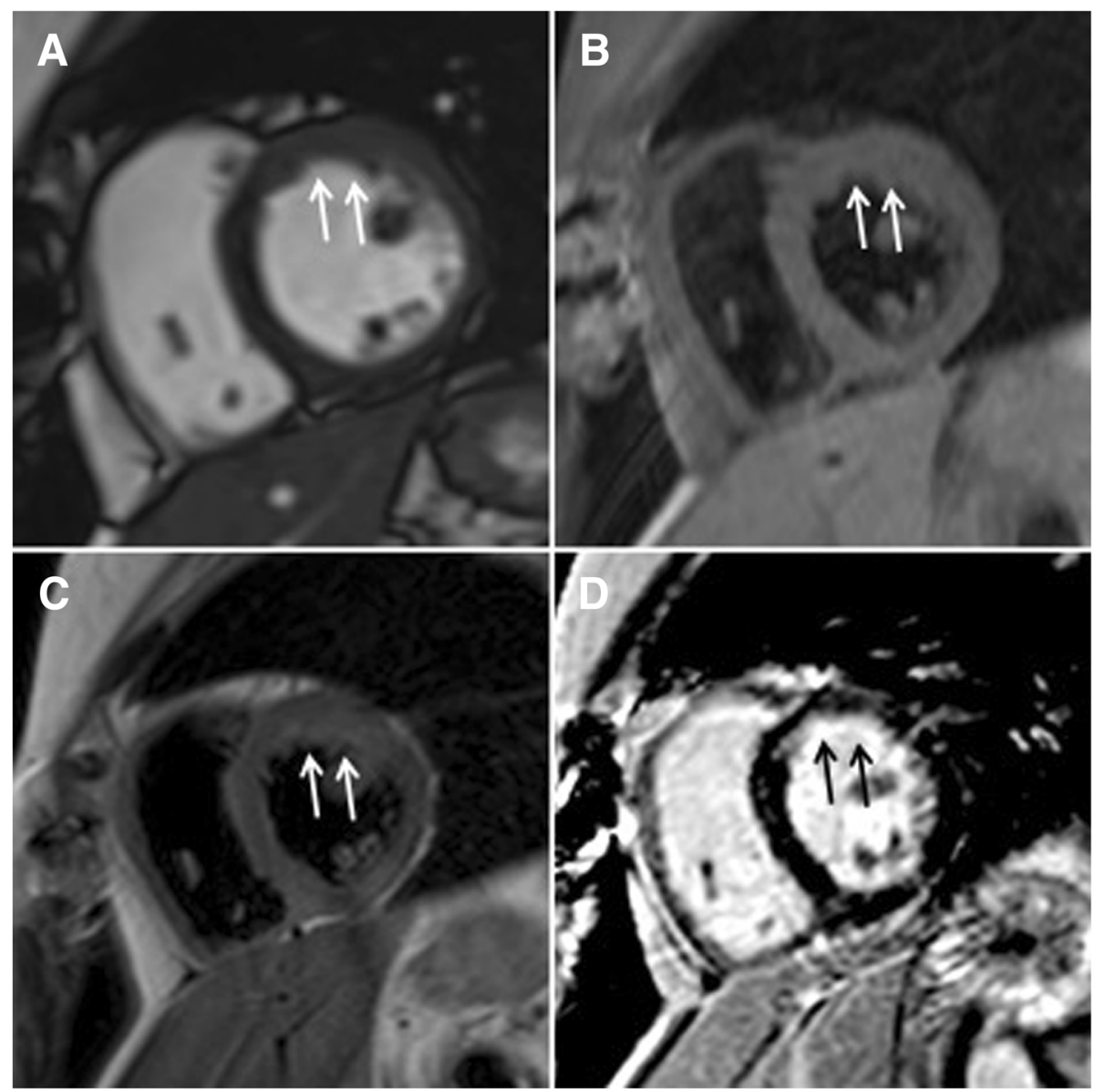

Fig. 4 The CMR study after 7 cycles of chemotherapy treatment. Complete response in cine (a), T1-weighted (b), T2-weighted (c) and LGE (d) images. $\mathrm{LGE}=$ late gadolinium enhancement

months from initial diagnosis the patient is still free of recurrence of tumor (Fig. 5) with no compromises of the quality of life.

\section{Discussion and conclusions}

Primary cardiac tumor is a very rear pathology with an incidence of less than $0.1 \%$. The incidence has increased over 3 eras (per 100 million persons): 25.1 in 1973 to 1989, 30.2 in 1990 to 1999, and 46.6 in 2000 to 2011. During those years the incidence of sarcomas and lymphomas increased and the incidence of mesotheliomas decreased [6, 7]. The patients can commonly present with dyspnea (48\%) as noticed in the case of our patient, chest pain $(22 \%)$, heart failure $(13 \%)$, and pericarditis (5\%) [8].

Most sarcomas grow very fast, and cause death through widespread infiltration of the myocardium, obstruction of blood flow through the heart, and/or distant metastases. Even with complete resection, most patients develop recurrent disease rapidly, thus according to different sources the median survival is typically 6 to 12 months [5]. The 32-year experience in Mayo clinic showed that the most common histological subtype of heart sarcomas was angiosarcoma (41\%) and median survival with complete resection was 17 months compared to 6 months with incomplete resection [9]. Due to poor results, different experimental techniques are being used. In China, Shanghai, 6 patients with non-metastatic primary cardiac sarcoma underwent cardiac transplantation, they also included other 40 patients after cardiac transplantation of the same reason found in literature. Among the 46 patients overall median survival was 16 months, ranging from 2 to 112 months, with the worst results in angiosarcoma group (9 vs 36 months). The patients with grade 2 sarcomas survived much longer than with grade 3 tumors (85 vs. 18 months) [10]. Auto-transplantation of the heart in the aim to perform a complete resection has also been attempted. Survival up to 5.5 years after complete surgical resection with the auto-transplantation technique has been reported [11]. Another successful cardiac autotransplantation procedure in the case of spindle cell sarcoma was reported in 

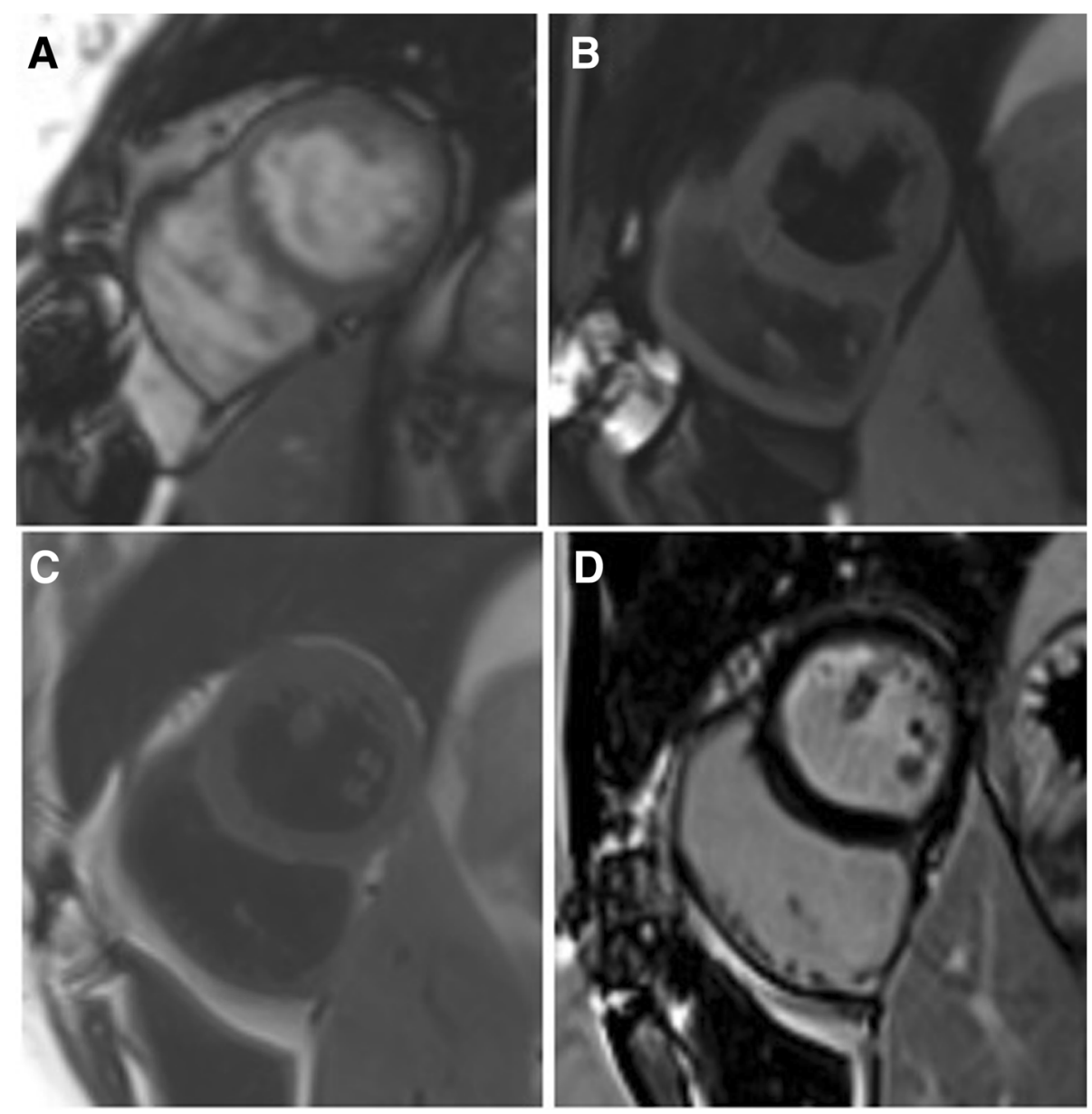

Fig. 5 CMR after 26 months from the diagnosis. Still in complete response in cine (a), T1-weighted (b), T2-weighted (c) and LGE (d) images

Japan. The tumor was excised completely together with most of the left atrium, which was the reconstructed using bovine pericardial patch. The patient was doing well 30 months after surgery [12].

UPSs are aggressive and locally invasive tumors, frequently making complete surgical excision unfeasible, which leads to a poor prognosis and a low survival rate [4]. Recently a similar case of 61-year-old woman with undifferentiated pleomorphic sarcoma of the left atrium was reported. The resection was incomplete, the patient presented with a relapse 6 months later. The outcome was much different. Anthracycline-based palliative chemotherapy was started, despite that the patient died 9 months after initial diagnosis [13]. However, radical surgical treatment can be successful and lead to good survival rate, e.g.:78-year-old woman with the UPS in left atrium underwent radical surgical resection and refused adjuvant chemotherapy. She survived 3 years and then presented with advanced metastatic disease and decompensated heart failure and died [14].
Neoadjuvant or adjuvant chemotherapy has been used to improve outcomes of the poor results with resection alone. Adjuvant chemotherapy with doxorubicin and ifosfamide is usually recommended postoperatively [11]. A study in France was conducted where effects of chemotherapy which included doxorubicin after surgical resection was analyzed. Median survival of 15 patients was 12 months, survival rate of 2 years was $26 \%$. Survival was significantly longer for patients with completely resected tumors (22 vs 7 months) and those who did not have angiosarcoma (18 vs 7 months). [15] Although all in all studies conclude that chemotherapy does not change the natural course of the disease there are a few cases in literature where multidisciplinary approach to different type primary cardiac sarcomas including chemotherapy has led to good survival rates [16-18]. A study from Cleveland Clinic where totally 42 patients with cardiac sarcoma treated from 1988 to 2013 were analyzed stated that multimodality therapy (any combination of surgery, radiation therapy, and chemotherapy) 
was associated with improved survival compared to surgery, radiation therapy of chemotherapy alone [3].

The case we presented is unique because without radical surgery and after chemotherapy the patient got into complete remission which lasts already 26 months. This already overcomes all median survival rates cited in literature even with the complete resection of tumor. We believe this is due to aggressive multidisciplinary approach.

The review is limited because the disease is very rare, the data are scarce, mostly case reports and experiences of single institutions are published. Therefore, individual approach to every case is extremely important and treatment options should be discussed thoroughly in the multidisciplinary team.

\section{Abbreviations}

CMR: Cardiovascular magnetic resonance; CT: Computed tomography; CTCAE: Common Terminology Criteria for Adverse Events; GCSF: Granulocyte-colony stimulating factors; IAS: Interatrial septum; LA: Left atrium; LDH: Lactate dehydrogenase; LV: Left ventricle; TTE: Transthoracic echocardiography; UPS: Undifferentiated pleomorphic sarcoma

\section{Acknowledgments}

Not applicable.

\section{Funding}

Not applicable.

\section{Availability of data and materials}

All data generated or analyzed during this study are included in this article.

\section{Authors' contributions}

$\mathrm{AV}^{1}$ prepared the data and also drafted the manuscript. LU prepared the data. EE revised the manuscript. DV treated the patient with chemotherapy and prepared the data. EE, AV $, A J, J P, A K, L P$ diagnosed the patient. EE, DV $E J, R J, L J, R Z$ and RB managed the treatment strategy. AS and RB performed the surgical treatment. $A V^{4}$ took care of the patient postoperativelly. EE and DV perform follow-up of this patient. AV', AJ, TL prepared CMR images and keeps evaluating further. All authors have read and approved the final manuscript.

\section{Ethics approval and consent to participate} Not applicable.

\section{Consent for publication}

Written informed consent was obtained from the patient for publication of this case report and any accompanying images.

\section{Competing interests}

The authors declare that they have no competing interests.

\section{Publisher's Note}

Springer Nature remains neutral with regard to jurisdictional claims in published maps and institutional affiliations.

\section{Author details}

${ }^{1}$ Department of Cardiology, Medical Academy, Lithuanian University of Health Sciences, Kaunas, Lithuania. ²Department of Oncology and Hematology, Medical Academy, Lithuanian University of Health Sciences, Kaunas, Lithuania. ${ }^{3}$ Department of Radiology, Medical Academy, Lithuanian University of Health Sciences, Kaunas, Lithuania. ${ }^{4}$ Department of Cardiac, Thoracic and Vascular Surgery, Medical Academy, Lithuanian University of Health Sciences, Kaunas, Lithuania. Institute of Cardiology, Lithuanian University of Health Sciences, Kaunas, Lithuania. ${ }^{6}$ Department of Pathology, Medical Academy, Lithuanian University of Health Sciences, Kaunas, Lithuania.
}

Received: 22 December 2018 Accepted: 13 May 2019

Published online: 31 May 2019

\section{References}

1. Barreiro M, Renilla A, Jimenez JM, Martin M, Al Musa T, Garcia L, et al. Primary cardiac tumors: 32 years of experience from a Spanish tertiary surgical center. Cardiovasc Pathol. 2013;22:424-7.

2. Munin MA, Goerner MS, Raggio I, Wisner J, Tettamanzi A, Godia J, et al. A rare cause of dyspnea: undifferentiated pleomorphic sarcoma in the left atrium. Cardiol Res 2017;8(5):241-245. doi: https://doi.org/10.14740/cr590w. Epub 2017 Oct 27

3. Randhawa JS, Budd GT, Randhawa M, Ahluwalia M, Jia X, Daw H. Primary cardiac sarcoma: 25-year Cleveland Clinic experience. Am J Clin Oncol. 2016; 39(6):593-9.

4. Vallés-Torres J, Izquierdo-Villarroya MB, Vallejo-Gil JM, CasadoDomínguez JM, Roche Latasa AB, Auquilla-Clavijo P. Cardiac Undifferentiated Pleomorphic Sarcoma Mimicking Left Atrial Myxoma. J Cardiothorac Vasc Anesth. 2018. pii: S1053-0770(18)30110-1. doi: https://doi.org/10.1053/j.jvca.2018.02.010.

5. Truong PT, Jones SO, Martens B, Alexander C, Paquette $M$, Joe $H$, et al. Treatment and outcomes in adult patients with primary cardiac sarcoma: the British Columbia Cancer Agency experience. Ann Surg Oncol. 2009;16:3358.

6. Lam KY, Dickens P, Chan AC. Tumors of the heart. A 20-year experience with a review of 12,485 consecutive autopsies. Arch Pathol Lab Med. 1993;117:1027.

7. Oliveira GH, Al-Kindi SG, Hoimes C, Characteristics PSJ. Survival of malignant cardiac tumors: a 40-year analysis of $>500$ patients. Circulation. 2015;132(25):2395-402. https://doi.org/10.1161/ CIRCULATIONAHA.115.016418 Epub 2015 Oct 14.

8. Isambert N, Ray-Coquard I, Italiano A, Rios M, Kerbrat P, Gauthier M, et al. Primary cardiac sarcomas: a retrospective study of the French sarcoma group. Eur J Cancer. 2014;50:128-36.

9. Kumar SK, Okuno SH, Schaff HV, Porrata LF, Buckner JC, Moynihan TJ. Malignant primary cardiac tumors: review of a single institution experience. Cancer. 2008;112(11):2440-6. https://doi.org/10.1002/cncr.23459.

10. Li H, Yang S, Chen H, Yang Z, Hong T, Hou Y, et al. Survival after heart transplantation for non-metastatic primary cardiac sarcoma. J Cardiothorac Surg 2016;11(1):145.

11. Palaskas N, Thompson K, Gladish G, Agha AM, Hassan S, Iliescu C, et al. Evaluation and Management of Cardiac Tumors. Curr Treat Options Cardiovasc Med. 2018;20(4):29. https://doi.org/10.1007/s11936-0180625-z.Review.

12. Suzuki K, Toda K, Saito S, Miyagawa S, Yoshikawa Y, Hata H. Autotransplantation for cardiac sarcoma with fenestrated patch and in situ pulmonary vein fixation. Circ J 2019 Feb 8. doi: https://doi.org/10.1253/circj. CJ-18-0680. [Epub ahead of print].

13. Watson R, Frye J, Trieu M, Yang MX. Primary undifferentiated pleomorphic cardiac sarcoma with MDM2 amplification presenting as acute left-sided heart failure. BMJ Case Rep. 2018;30:2018. https://doi. org/10.1136/bcr-2018-226073.

14. Zapata Laguado MI, Palacios Rojas JO, Gómez Lopez EA, Velasco Morales ML, Orozco de la Hoz CE. High-grade left atrial pleomorphic sarcoma: case report and review. Case Rep Oncol. 2017;10(3):1138-43. https://doi.org/10. 1159/000485561.eCollection2017Sep-Dec.

15. Llombart-Cussac A, Pivot X, Contesso G, Rhor-Alvarado A, Delord JP, Spielmann $M$, et al. Adjuvant chemotherapy for primary cardiac sarcomas: the IGR experience. Br J Cancer. 1998 Dec;78(12):1624-8.

16. Pessotto R, Silvestre G, Luciani GB, Anselmi M, Pasini F, Santini F, et al. Primary cardiac leiomyosarcoma: seven-year survival with combined surgical and adjuvant therapy. Int J Cardiol. 1997;60:91.

17. GM M, Reardon MJ, Haas J, Lazar J, Hindenburg A. A combined modality approach to recurrent cardiac sarcoma resulting in a prolonged remission: a case report. Chest. 2003;123:1766.

18. T N, Fukuda T, Suzuki T, Kaneko T, Morikawa Y. Primary cardiac angiosarcoma: 53 months' survival after multidisciplinary therapy. Ann Thorac Surg. 1997;63:1160. 